\title{
Magnetic Field Alignment of Randomly Oriented, High Aspect Ratio Silicon Microwires into Vertically Oriented Arrays
}

\author{
Joseph A. Beardslee, Bryce Sadtler, and Nathan S. Lewis* \\ Beckman Institute and Kavli Nanoscience Institute, Division of Chemistry and Chemical Engineering, 127-72, 210 Noyes Laboratory, \\ California Institute of Technology, Pasadena, California 91125, United States
}

\begin{abstract}
External magnetic fields have been used to vertically align ensembles of silicon microwires coated with ferromagnetic nickel films. X-ray diffraction and image analysis techniques were used to quantify the degree of vertical orientation of the microwires. The degree of vertical alignment and the minimum field strength required for alignment were evaluated as a function of the wire length, coating thickness, magnetic history, and substrate surface properties. Nearly $100 \%$ of $100 \mu \mathrm{m}$ long, $2 \mu \mathrm{m}$ diameter, $\mathrm{Si}$ microwires that had been coated with $300 \mathrm{~nm}$ of Ni could be vertically aligned by a $300 \mathrm{G}$ magnetic field. For wires ranging from 40 to $60 \mu \mathrm{m}$ in length, as the length of the wire

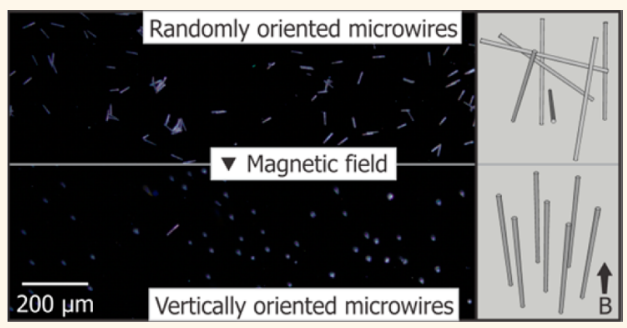
increased, a higher degree of alignment was observed at lower field strengths, consistent with an increase in the available magnetic torque. Microwires that had been exposed to a magnetic sweep up to $300 \mathrm{G}$ remained magnetized and, therefore, aligned more readily during subsequent magnetic field alignment sweeps. Alignment of the Ni-coated Si microwires occurred at lower field strengths on hydrophilic Si substrates than on hydrophobic Si substrates. The magnetic field alignment approach provides a pathway for the directed assembly of solution-grown semiconductor wires into vertical arrays, with potential applications in solar cells as well as in other electronic devices that utilize nano- and microscale components as active elements.
\end{abstract}

KEYWORDS: magnetic coatings · vapor-liquid-solid growth $\cdot \mathrm{Ni}$ electrodeposition $\cdot \mathrm{X}$-ray diffraction $\cdot$ image analysis

S ilicon microwire array solar cells have been shown to yield energy conversion efficiencies as high as $7.9 \%$, while using less Si than conventional planar cells. ${ }^{1-4}$ In addition to the promise of scalable production, such microwire arrays also increase the physical flexibility of the resulting device. ${ }^{5}$ Vertical alignment of the microwires decouples the directions of light absorption and minority carrier charge collection and can thus provide excellent performance, provided that the microwires are sufficiently long and are oriented appropriately to attain $96 \%$ peak absorption of incident light. ${ }^{6}$

Current Si microwire array solar cell designs use a series of clean room processes, such as photolithography and metal evaporation, to obtain and retain the preferred, predominantly vertical, orientation of the microwires. For many materials, epitaxial growth of vertically oriented microwires on a substrate, and removal of the wires from the substrate while retaining the vertical alignment of the microwire array, is not possible or economical. Colloidal methods have been used to synthesize high aspect ratio nano- and microwires composed of metal oxide, ${ }^{7}$ metal chalcogenide, ${ }^{8}$ groups $\mathrm{III}-\mathrm{V},{ }^{9}$ and elemental semiconductor materials. ${ }^{10}$ These processes produce a randomly oriented solution of particles, but the scalability of such processes could be leveraged if a self- or directed-assembly process could be developed that would align the randomly oriented suspension of microwires into the desired vertically oriented microwire array.

Self- and directed-assembly have been well-documented on the nanoscale, and objects ranging in aspect ratio from disks to rods have been assembled using methods that include electric- and magnetic-fieldassisted assembly, ${ }^{11-13}$ Langmuir-Blodgett assembly, ${ }^{14}$ and evaporation-induced *Address correspondence to
nslewis@caltech.edu.

Received for review September 10, 2012 and accepted October 20, 2012.

Published online October 20, 2012 10.1021/nn304180k

C 2012 American Chemical Society 
self-assembly. ${ }^{15-18}$ Such methods have been used to create well-ordered arrays of a variety of metallic, semiconducting, and magnetic colloidal nanoparticles. However, as the size of the particle increases to the microscale, as would be required for $\mathrm{Si}$ and some other indirect band gap semiconductors, Brownian motion becomes insufficient to overcome the kinetic barriers to rotation. Low aspect ratio structures (aspect ratio $\leq 1$ ), such as spheres and platelets, can nevertheless be readily assembled through the use of surface energy interactions ${ }^{19,20}$ or externally applied fields. ${ }^{21-23} \mathrm{How}$ ever, for high aspect ratio structures, such as wires with lengths of $10 \mu \mathrm{m}$ or greater, the tendency toward aggregation typically precludes the use of methods that rely on surface interactions or evaporative assembly. Therefore, additional input energy is required, which can be applied in the form of external fielddriven directed-assembly. Directed-assembly techniques, such as electric, magnetic, or fluidic alignment, have been used to prepare horizontally oriented arrays of wire structures. ${ }^{24-28}$ Extending these field alignment approaches to produce vertically oriented arrays would enable the fabrication of wire array solar cells without the need for the use of a clean room.

We demonstrate herein that 40-100 $\mu \mathrm{m}$ long, 1-2 $\mu \mathrm{m}$ diameter, Si microwires coated with a thin layer of ferromagnetic Ni can be aligned vertically into a monolayer, over areas on the square centimeter scale, by the use of magnetic fields. These vertically oriented $\mathrm{Si}$ microwire arrays have been subsequently captured in a polymer film to produce flexible, polymer-embedded, vertically oriented arrays of Si microwires. The degree of vertical alignment and the minimum field strength required for alignment have been evaluated as a function of the length, coating thickness, and magnetic field history of the Ni-coated Si microwires, as well as the substrate surface properties.

\section{RESULTS}

Sample Preparation. Wire Growth. Figure 1A shows Si microwire arrays that had been grown uniformly over large areas $\left(\sim 1 \mathrm{~cm}^{2}\right)$ and conformally coated with electrodeposited Ni. Vapor-liquid-solid (VLS)-grown Si microwires were chosen as a model system because the wire length and diameter can be controlled by variation of the growth parameters (deposition time and wire template hole diameter, respectively). ${ }^{29}$ Samples with lengths ranging from 40 to $100 \mu \mathrm{m}$ (measured prior to removal from the growth substrate, with a typical length variation of $\pm 2 \mu \mathrm{m}$ ) were tested. The inset in Figure 1 shows a cross section of the conformal coating of $\mathrm{Ni}$ on one microwire. Figure $1 \mathrm{~B}$ presents a plot of superconducting quantum interference device (SQUID) data obtained for a similar $\mathrm{Ni}$-coated array, with the magnetic saturation $\left(M_{\mathrm{S}}\right)$ at the hysteresis curve maximum $\left(0.65 \mathrm{~A} \mathrm{~m}^{-1}\right)$
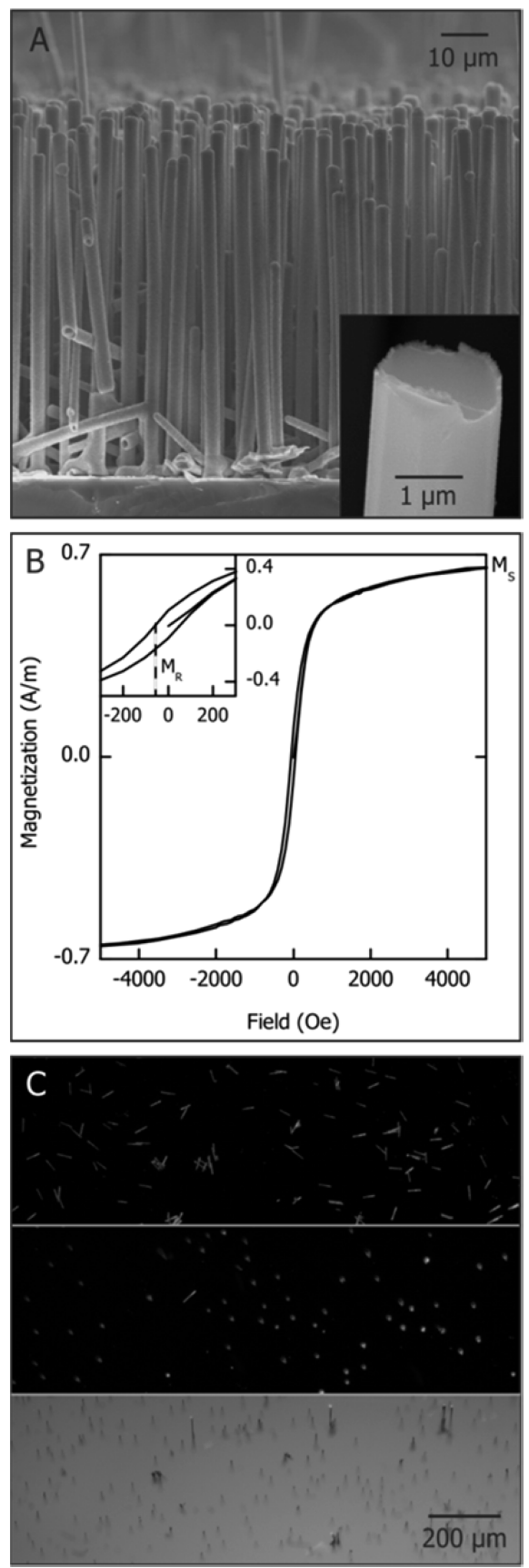

Figure 1. (A) Scanning electron micrograph of VLS-grown Si microwires coated with $\mathrm{Ni}$ by electrodeposition in Ni sulfamate solution (Transene) at $-1 \mathrm{~V}$ vs $\mathrm{Ag} / \mathrm{AgCl}$. (Inset) Cross section of a $\mathrm{Si}$ microwire showing the thickness of the deposited Ni. (B) SQUID data for a Ni-plated wire array, with the inset showing an expansion of the low-field region of the hysteresis curve. The magnetic saturation $\left(M_{\mathrm{S}}\right)$ and remnance $\left(M_{R}\right)$ are labeled. (C) Dark-field optical micrographs of horizontal microwires (top), vertically oriented microwires (center), and vertical microwires embedded in a PDMS film (bottom).

and a magnetic remnance $\left(M_{\mathrm{R}}\right)$ of $60 \mathrm{emu} \mathrm{cm}^{-3}$. Figure $1 \mathrm{C}$ displays an optical micrograph that shows representative results of the Ni plating process, with the top panel showing randomly deposited microwires oriented horizontally on a Si substrate, the 

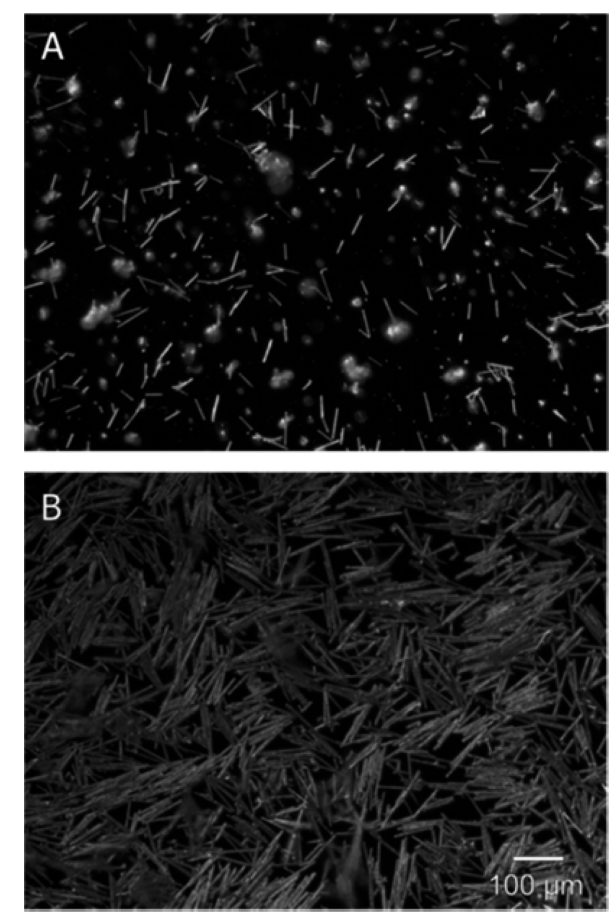

Figure 2. Dark-field optical micrographs of Ni-coated $\mathrm{Si}$ wires deposited on Si substrates using Langmuir-Blodgett deposition for sparse (A) and dense (B) films of horizontal wires. The scale bar of $100 \mu \mathrm{m}$ applies to both of the frames.

center panel showing microwires oriented vertically in the presence of an out-of-plane magnetic field, and the bottom panel showing vertically oriented microwires that had been captured in a polydimethylsiloxane (PDMS) film.

Wire Deposition. Figure 2 shows Si microwires that had been deposited onto clean Si substrates, using the dipper functionality of a Langmuir-Blodgett trough. Panel A depicts wires that had been deposited sparsely, whereas panel $B$ shows the same Si wires deposited into a closely packed horizontal layer. Panel $B$ is the result of the transfer of a film at the maximum of horizontal packing attainable at the surface of the trough without the layer buckling.

Particle Analysis. Figure 3 shows the results of image analysis of a typical vertically aligned Si microwire array sample. Panel A shows the raw dark-field optical micrograph. Panels $B$ and $C$ show the wires sorted by circularity into horizontal and vertical wire masks, respectively. This sample exhibited an alignment of $96 \%$ vertical. The lateral particle-to-particle distance was calculated by ImageJ image processing software to be $66 \mu \mathrm{m}$.

X-ray Diffraction. X-ray diffraction data were acquired for vertically aligned Si microwire array samples, and the data were compared to the predicted position for the $\mathrm{Si}\langle 111\rangle$ peak (Figure 4). The magnetically aligned $\mathrm{Si}$ microwire array sample (originally deposited on a $\mathrm{Si}$ surface roughened by anodic etching) showed a peak at the expected Si $\langle 111\rangle$ position. The peak for the
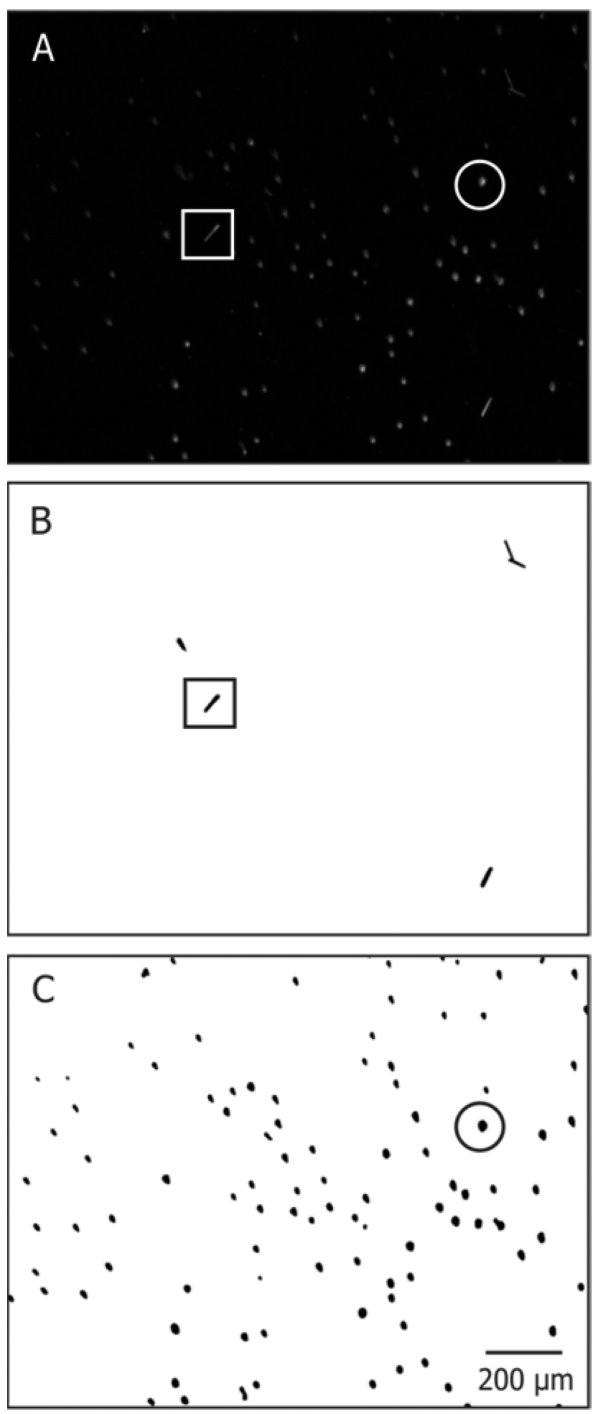

Figure 3. Image analysis process used to determine the average wire-to-wire distance as well as the vertical alignment percentage. (A) Dark-field optical micrograph of vertically aligned microwires. Masks were generated of the horizontally oriented (B) and vertically oriented (C) wires by sorting by circularity using the ImageJ particle analysis package. The scale bar of $200 \mu \mathrm{m}$ applies to all three frames.

magnetically aligned, vertically oriented Si microwire array had $97 \%$ of the integrated area within $\pm 5^{\circ}$ of the nominal position expected for a fully vertically oriented Si microwire array.

Alignment Plots. Alignment versus Coating Thickness. Figure 5 shows optical micrographs of Si microwires having between 20 and $300 \mathrm{~nm}$ thick coatings of sputtered Ni. Panel F presents the degree of alignment for each sample under a $2.5 \mathrm{kG}$ magnetic field, as a function of the thickness of the Ni film. The samples with a thin Ni coating showed nearly zero alignment, whereas those with a $300 \mathrm{~nm}$ coating of Ni were nearly completely aligned vertically by the magnetic field.

Alignment versus Magnetic Field. Figure 6 shows the fraction of vertically aligned Ni-coated Si microwires as the magnetic field strength was swept from 


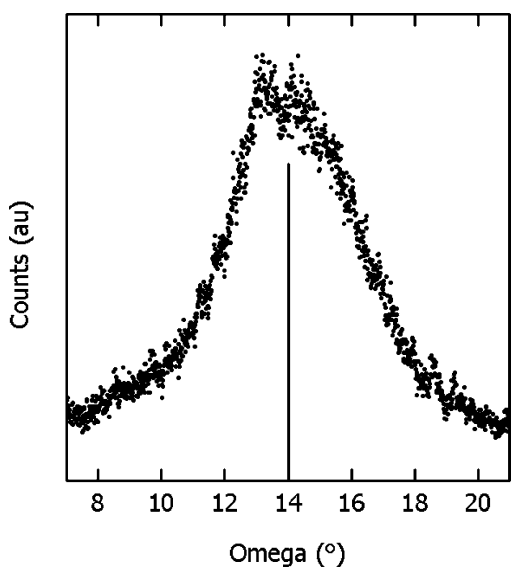

Figure 4. X-ray diffraction rocking curve for magnetically aligned, Ni-coated, Si microwires; $97 \%$ of the wires were within $\pm 5^{\circ}$ of substrate normal, indicated by the vertical line in the figure.
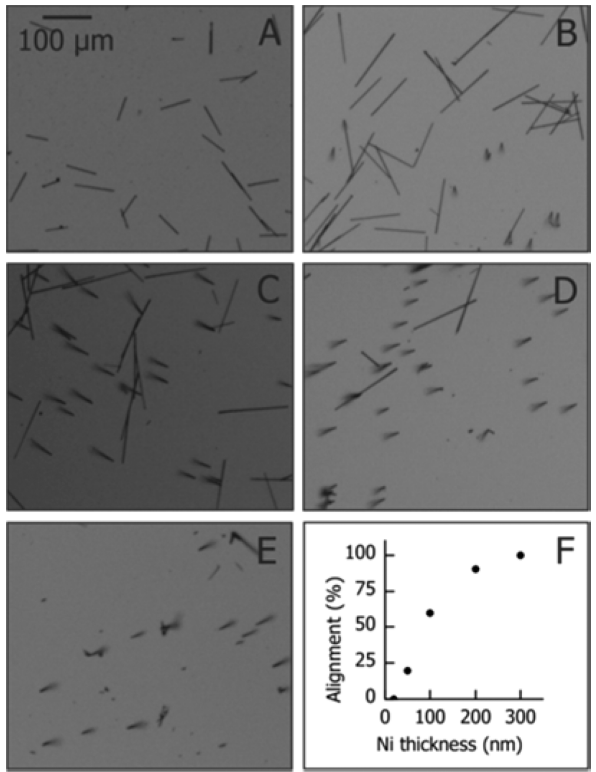

Figure 5. (A-E) Bright-field optical micrographs of Si microwires having $\mathrm{Ni}$ coatings of thickness varying from 20 to $300 \mathrm{~nm}$. (F) Plot of the vertical alignment percentage of the $\mathrm{Ni}$-coated $\mathrm{Si}$ microwires a function of the Ni coating thickness. The scale bar of $100 \mu \mathrm{m}$ applies to all of the frames.

0 to $350 \mathrm{G}$ at $\sim 5 \mathrm{G} \mathrm{s}^{-1}$. Samples that had been aligned in this manner showed a pronounced dependence of the degree of microwire alignment on the applied field strength, with longer microwires aligning more completely, and at lower field strengths, in response to the magnetic field. Panel B shows the dependence of the degree of alignment on the magnetic field history of the sample, indicating that the microwires aligned at lower field strengths during a second sequential sweep of the magnetic field than during an initial magnetic field sweep. The percentage of wire alignment observed in response to a third magnetic field sweep was nearly identical to that obtained in response to a second magnetic field sweep.

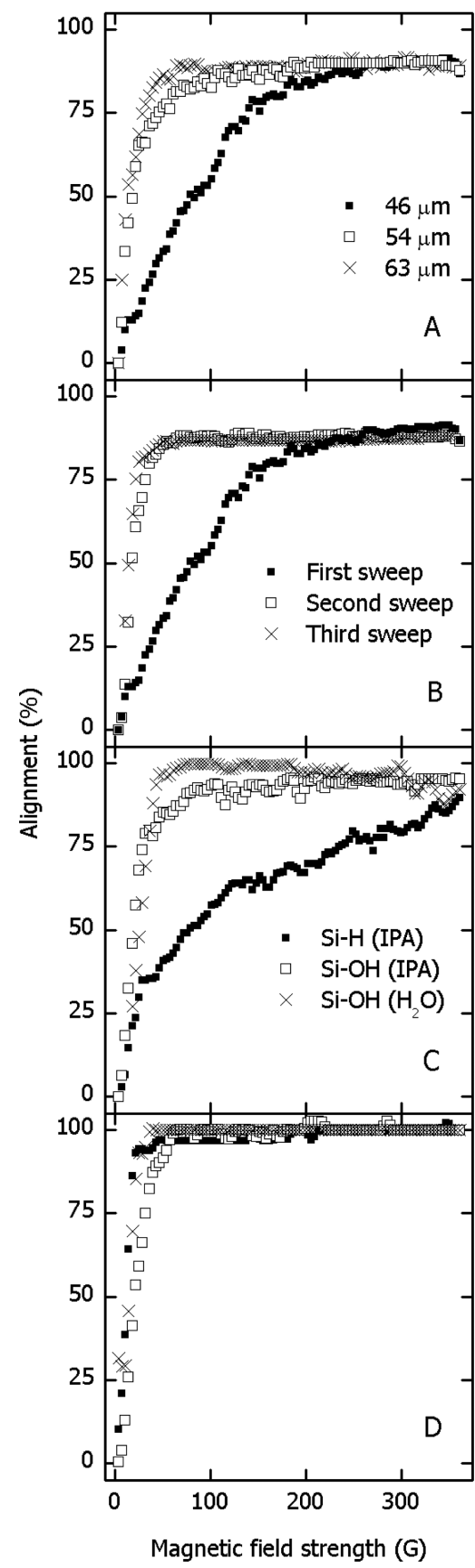

Figure 6. Percentage of vertically aligned $\mathrm{Ni}$-coated $\mathrm{Si}$ microwires as a function of applied magnetic field. (A) Magnetic alignment of microwires with varying length. (B) Degree of alignment for varying number of field sweeps. (C) Alignment dependence on the surface energy of the alignment substrate and suspending solvent. (D) Reproducibility between single sweep measurements for a typical sample.

Panel $\mathrm{C}$ demonstrates the impact of the substrate surface energy on the alignment of the Ni-coated $\mathrm{Si}$ microwires. A hydrophilic Si substrate clearly led to alignment of the microwires at lower field strengths than was observed when a hydrophobic Si substrate was used. Panel $D$ shows three individual runs plotting overlapping traces. The traces in panels $A, B$, and $C$ represent the average of four individual runs. 


\section{DISCUSSION}

Degree of Ordering. VLS-grown Si microwires exhibit good radial $( \pm 0.1 \mu \mathrm{m})$ and length $( \pm 2 \mu \mathrm{m})$ uniformity over large areas, making them well-suited as a model system to study the effects of magnetic alignment on microwire-type structures. To facilitate investigation of the approach, a conformal coating of $\mathrm{Ni}$ was applied to the Si microwires to provide a ferromagnetic handle on the Si (Figure 1). The coating was very uniform over the length of the wire, as verified by scanning electron microscope (SEM) cross sections at various heights along the wire array. SQUID data yielded a magnetic saturation of $0.65 \mathrm{~A} \mathrm{~m}^{-1}$, consistent with the presence of a thin layer of $\mathrm{Ni}$ on the Si microwires. The measured magnetic remnance $\left(60 \mathrm{emu} \mathrm{cm}^{-3}\right.$ at $300 \mathrm{~K}$ ) indicates that the wires will maintain a low amount of magnetism after exposure to the magnetic field. This magnetic history causes the wires to respond more readily to low field strengths on subsequent exposures (Figure 6). Figure $1 \mathrm{C}$ shows the result of Ni plating onto the Si microwires and provides definitive evidence that application of a magnetic field allowed an ensemble of horizontally oriented $\mathrm{Ni}$-coated Si microwires to rapidly shift to a vertical orientation.

Because the wires maintained their lateral ordering when the magnetic field was applied, control over the initial packing density allowed for control over the density of the final vertically oriented $\mathrm{Si}$ microwire ensemble (Supporting Information Figure 1). In solution, the microwires magnetically attracted each other and formed clumps. In contrast, magnetic alignment on a substrate produced a monolayer of microwires with a controllable packing density. The samples prepared herein were on the order of $1 \mathrm{~cm}^{2}$ in lateral area, with the area of alignment presumed to be limited by the equipment size, rather than by any inherent limit to scalability of the process.

The omega rocking curve data indicated that the magnetically aligned Si microwire ensembles were largely oriented vertically (Figure 4) because the observed peak corresponded well with the Si $\langle 111\rangle$ direction along the long axis of the wires. The sample shown was clearly well-oriented, as $97 \%$ of the integrated area corresponded to wires aligned at angles within $\pm 5^{\circ}$ of the substrate normal. Such an orientation is sufficiently vertical for wire arrays to have a long enough path length to absorb most incident sunlight. The small angular dispersion relative to that expected for a fully oriented microwire array is expected to produce scattering effects, which have been shown to enhance the optical absorption in other high aspect ratio array microwire assemblies. ${ }^{30-33}$

Effects of Microwire Dimensions, Coating Thickness, and Substrate Interactions. Alignment versus Coating Thickness. The percentage of vertically aligned microwires showed a smooth, monotonic increase versus the thickness of the applied Ni coating. Image analysis of optical micrographs was used to calculate the fraction of microwires that were aligned at each field strength. At the field strengths investigated, the samples showed virtually no alignment with a $20 \mathrm{~nm}$ thick $\mathrm{Ni}$ coating but showed a full vertical alignment with a $300 \mathrm{~nm}$ thick Ni coating. A step function dependence on thickness, with the entire ensemble of wires going from horizontal to vertical as the critical field strength was reached, was not observed, presumably due to small variations in the thickness of the $\mathrm{Ni}$ film within a batch of Ni-coated Si microwires. Because full vertical alignment is desired for many potential applications, this dependence allows fabrication of responsive wires with a minimum thickness of ferromagnetic coating.

Alignment versus Magnetic Field. The alignment percentage increased with an increase in the applied magnetic field (Figure 6). The traces in Figure 6 show data for microwire samples in solvent, suspended in the center of an electromagnet, under a CCD video camera. Panels $\mathrm{A}-\mathrm{C}$ are plotted with traces that were averaged from four individual runs, while panel $D$ shows individual runs, demonstrating the repeatability of the measurements. Separate runs are shown to exhibit the reproducibility that was generally observed in the alignment process. Panel A demonstrates that the alignment process occurred at lower field strengths as the length of the wire was increased, due to a higher available magnetic torque.

Panel B illustrates the result of a second sweep and indicates that the alignment process occurred at lower field strengths under such conditions, due to a combination of remnant magnetization in the wires as well as due to a decrease in surface energy interactions caused by delamination of the microwires from the substrate during the first field sweep. Additional sweeps traced the path of the second sweep.

Panel $\mathrm{C}$ demonstrates the role of surface energy interactions, with two samples of Ni-coated microwires prepared in the same batch and both aligned in isopropyl alcohol, using the same procedure, but with one alignment performed on a low surface energy $\mathrm{Si}-\mathrm{H}$-terminated $\mathrm{Si}$ surface and with the other on a high surface energy $\mathrm{Si}-\mathrm{OH}$-terminated $\mathrm{Si}$ surface. The more hydrophilic $\mathrm{Si}-\mathrm{OH}$ surface produced more facile alignment of the Ni-coated Si microwires, presumably due to a more favorable interaction with the solvent and a lessening of the hydrophobic attraction between the substrate and microwires. Similarly, microwires aligned on a $\mathrm{Si}-\mathrm{OH}$ surface in $\mathrm{H}_{2} \mathrm{O}$ responded to a lower magnetic field strength than microwires aligned on a $\mathrm{Si}-\mathrm{OH}$ surface in isopropyl alcohol, due to a more favorable substrate-solvent interaction.

A variety of solvents can be used if the appropriate balance with the substrate surface energy is met, allowing for the alignment of wires within a prepolymer solution. Using this method, wires have been captured 
in flexible PDMS films and removed from the substrate (Figure 1C), similar to other literature reports. ${ }^{31} \mathrm{Re}-$ moval from the substrate is a key step that allows for the integration of a vertical wire film into a device. Additionally, if a conductive polymer is used, it can make ohmic contact to the $\mathrm{Ni}$ film and serve as the back contact for the device.

Device Design. The image analysis of a typical sample (Figure 3) showed good vertical alignment of $96 \%$. Analysis of the masked image with only the vertical microwires displayed (C) gave an average particleto-particle distance of $66 \mu \mathrm{m}$, corresponding to a sparse ensemble. This distance can be tuned by modifying the original packing fraction of the horizontally deposited film (Figure 2). The packing fraction of the microwires will impact the light absorption properties of an array and, subsequently, its performance as a device, so the ability to increase the density to maximize absorption while using a minimum of material is a valuable control parameter.

Previous studies have shown that $\mathrm{Si}$ microwire arrays can exhibit light absorption efficiencies over $90 \%$ across the visible spectral region, ${ }^{31}$ using arrays with a packing fraction of approximately $5 \%$, corresponding to a $7 \mu \mathrm{m}$ microwire-to-microwire distance. The arrays prepared in this work had a lower packing fraction, which will likely negatively impact light absorption. Tuning the packing fraction by obtaining higher horizontal densities on the substrate is therefore expected to be an important step in progress toward a functional, magnetically aligned, light-absorbing film. The ultimate density that can be achieved is limited by the agglomerization produced by magnetic dipole coupling between nearby microwires, which depends on the geometry of the microwires as well as their surface chemistry. If the horizontal film is too dense, physically adjacent microwires will tend to couple together when aligning vertically, leading to a lower quality film.

The Ni coating was intended primarily to serve as a ferromagnetic handle but might also have utility in future device designs. In systems with appropriate band offsets, for example $\mathrm{p}$-Si, Ni can form an ohmic contact to the wire and could make the back contact for a Si microwire-based solar cell. In this case, the Ni could by removed asymmetrically by use of a polymer masking step ${ }^{2}$ to expose the $\mathrm{Si}$ on the top half of the wire. Removal of the Ni shell on the top half of the wire with a commercially available etchant would allow formation of a rectifying liquid junction on the exposed portion of the Si microwire.

Vertically aligned microwires were captured in flexible PDMS films and removed from the alignment substrate (Figure 1C), similar to other reports in which denser arrays of Si wires were removed from the Si growth substrates. ${ }^{31}$ The film was prepared by alignment of the wires in a PDMS prepolymer solution. This procedure allowed the vertical pattern of the wires to be retained in the polymer film after the magnetic field was removed. Removal of the wires from the substrate enables the possibility of processing of a stand-alone vertical film into the active layer of a solar device. Additionally, this step provided for reuse of the alignment substrate.

\section{CONCLUSIONS}

Ensembles of micrometer-sized Si wires with ferromagnetic Ni coatings can be oriented vertically using magnetic fields. The vertical and lateral ordering of these ensembles have been characterized using X-ray diffraction and optical image analysis. The degree of, and minimum field strength for, vertical alignment depended on the length of the microwires as well as on the thickness of the Ni coating. Longer microwires experienced more magnetic torque and thus aligned at lower field strengths than shorter microwires. For a given microwire length, thicker $\mathrm{Ni}$ coatings enabled a higher percentage of vertical alignment at a given magnetic field strength. Additionally, the surface interactions of the substrate and solvent could be tuned to produce more complete and facile alignment of the microwires. Microwire ensembles with vertical orientations in excess of $97 \%$ within $\pm 5^{\circ}$ of normal were achieved over square centimeter scale areas, and the oriented microwires were successfully embedded into polymer membranes while retaining the orientation of the microwires in the array. The magnetic alignment of micrometer scale $\mathrm{Si}$ wires has been demonstrated; however, this procedure should be equally applicable to the assembly of high aspect ratio nanowires and nanorods. This method is of interest for the cleanroom-free fabrication of flexible, array-based solar and electronic devices, in which preferential orientation of microwire-type objects is desired and useful.

\section{EXPERIMENTAL SECTION}

Fabrication. Microwire Growth. Crystalline Si microwires with lengths ranging from 40 to $100 \mu$ m were fabricated via the VLS growth technique using a chemical vapor deposition reactor. ${ }^{29}$ $\mathrm{Cu}$-catalyzed microwires were grown at $1000^{\circ} \mathrm{C}$ using $\mathrm{SiCl}_{4}$ as a precursor, $\mathrm{BCl}_{3}$ as a p-type dopant, and $\mathrm{H}_{2}$ as a carrier gas (see Supporting Information).
For most samples, Ni was deposited on the wires electrochemically to produce a ferromagnetic handle for alignment. The Si microwire arrays were first etched in buffered hydrofluoric acid (Transene, used as received), immediately prior to electrodeposition of the $\mathrm{Ni}$ films. A commercial boric-acidbuffered Ni sulfamate solution (Transene, used as received) served as the electroplating bath. All of the $\mathrm{Ni}$ films were 
deposited on p-type (resistivity $<0.005 \Omega \cdot \mathrm{cm}$ ) Si microwires at room temperature in a stirred, three-electrode electrochemical cell, at $-1 \mathrm{~V}$ vs a $\mathrm{Ag} / \mathrm{AgCl}$ reference electrode, with a Pt foil serving as the counter electrode. The thickness of the Ni coating was systematically varied from 100 to $500 \mathrm{~nm}$ by adjusting the deposition time from 5 to $15 \mathrm{~min}$. The resulting $\mathrm{Ni}$ film thickness depended linearly on the growth time. For some of the samples, Ni was sputtered from a $99.999 \%$ pure target (Kurt J. Lesker, used as received) using a home-built RF magnetron sputter deposition system. In the sputtering process, Ni was deposited at $90 \mathrm{~W}$ forward power in an atmosphere of $1 \mathrm{mTorr}$ Ar, from a base pressure of $<1 \times 10^{-7}$ Torr, with resulting coating thicknesses between 20 and $300 \mathrm{~nm}$.

Horizontal Deposition of Si Microwires. The Si microwires were deposited horizontally onto $1 \mathrm{~cm}^{2}$ Si substrates using a KSV Instruments Langmuir-Blodgett trough equipped with a dipper. Suspensions of wires in isopropyl alcohol $(\sim 10-$ $100 \mathrm{mg} \mathrm{mL}^{-1}$ ) were deposited on a water subphase. Horizontal films of microwires that had different packing densities were produced by varying the trough compression. The microwires were deposited on smooth, single-crystal Si substrates. The substrates were rendered hydrophobic by a 2 min etch in buffered hydrofluoric acid (Transene, used as received) or hydrophilic by the same hydrofluoric acid etch followed by a UV/ozone treatment for $30 \mathrm{~min}$ in a ProCleaner UV/ozone system. The substrates were degreased with isopropyl alcohol prior to treatment. Some substrates were roughened by anodic etching in an ethanolic solution of hydrofluoric acid (1:2:3 buffered hydrofluoric acid, ethanol, and deionized water by volume) for $60 \mathrm{~min}$ at a constant current density of $11.5 \mathrm{~mA} \mathrm{~cm}^{-2}$.

Magnetic Alignment. With the exception of the films shown in Figure 5, which were prepared using a $2.5 \mathrm{kG}$ permanent magnet, wires were aligned in the center of a liquid-cooled solenoid electromagnet (fabricated in-house) that had field strengths that varied from $1 \mathrm{G}$ to $1.5 \mathrm{kG}$. The field direction was oriented along the substrate normal. The electromagnet was installed below the objective of an Olympus BX-51 microscope that was equipped with a CCD camera that recorded video at 5 frames per second. A Keithley source meter controlled by LabTracer software was used to power the magnetic field sweep. The magnetic field strength was calibrated to the power applied to the electromagnet using a Bell 5180 gaussmeter, with magnetic fields stable to within $\pm 1 \mathrm{G}$ over the course of a typical experiment. Ramp rates of $\sim 5 \mathrm{G} \mathrm{s}^{-1}$ were used in most experiments. The sweeps were performed sufficiently slowly that the wires exhibited a steady-state alignment response at each value of the magnetic field strength. This behavior was achieved by increasing the sweep time until the shape of the trace remained the same.

To obtain vertically aligned Si microwire arrays embedded in polymer films, Sylgard 184 (Dow Corning, used as received) was dissolved in dichloromethane $(\sim 10 \% \mathrm{v} / \mathrm{v})$. The Si microwires were aligned using the polydimethylsiloxane prepolymer solution as the solvent, the polymer was cured, typically at room temperature, and the magnetic field was removed.

Characterization. Microwire Fabrication. The dimensions of the wires were measured using a ZEISS 1550 VP field emission scanning electron microscope. Cross sections of the as-grown arrays were imaged before and after deposition, and the length and width measurements were obtained by averaging over 5-10 wires per sample. The magnetic properties of the microwire arrays were characterized using a Quantum Design superconducting quantum interference device. Hysteresis curves of $\mathrm{Ni}$-coated wire arrays were obtained at $300 \mathrm{~K}$ from -8 to $8 \mathrm{~T}$ and were normalized to the deposited weight of $\mathrm{Ni}$.

Image Analysis. The lateral ordering of vertically aligned $\mathrm{Si}$ microwires was measured by image analysis of optical micrographs. Optical micrographs were imported into the image analysis software ImageJ, ${ }^{34}$ which converted the micrographs into binary black and white images. The ImageJ particle analysis package was used to analyze the binary image as well as to separate it by particle circularity into two masks, one of horizontally oriented wires and one of vertically oriented wires. The average particle-to-particle spacing was calculated from the coordinates of the wires in the vertical image. This technique was applied to individual images as well as to still frames that had been captured from the video imagery.

$X$-ray Diffraction. X-ray diffraction was used to quantify the degree of vertical orientation of the magnetically aligned microwire arrays. Omega rocking curves were obtained using a Cu LFF source with an acquisition time of $15 \mathrm{~min}$ at $1.8 \mathrm{~kW}$, on a four-axis PANalytical X'Pert X-ray diffractometer. The width of the Si (111) diffraction peak, corresponding to the crystal planes perpendicular to the long growth axis of the microwires, was characteristic of the vertical alignment variation from the substrate normal. ${ }^{35}$ The vertical alignment was quantified by the percentage of wires within $\pm 5^{\circ}$ of the substrate normal, as measured by the integrated area under the curve. The size of the incident beam was sufficiently large to analyze the entire $1 \mathrm{~cm}^{2}$ area of the sample.

Conflict of Interest: The authors declare no competing financial interest.

Acknowledgment. This work was supported by the DOE "Light-Material Interactions in Energy Conversion" Energy Frontier Research Center under Grant DE-SC0001293. Research was in part carried out at the Molecular Materials Research Center of the Beckman Institute of the California Institute of Technology. B.S. acknowledges the Beckman Institute of the California Institute of Technology for a postdoctoral fellowship.

Supporting Information Available: Parameters and procedures for vapor-liquid-solid growth of Si microwires and a figure depicting the lateral retention of order of microwires during magnetic alignment. This material is available free of charge via the Internet at http://pubs.acs.org.

\section{REFERENCES AND NOTES}

1. Boettcher, S. W.; Spurgeon, J. M.; Putnam, M. C.; Warren, E. L.; Turner-Evans, D. B.; Kelzenberg, M. D.; Maiolo, J. R.; Atwater, H. A.; Lewis, N. S. Energy-Conversion Properties of Vapor-Liquid-Solid-Grown Silicon Wire-Array Photocathodes. Science 2010, 327, 185-187.

2. Kelzenberg, M. D.; Turner-Evans, D. B.; Putnam, M. C.; Boettcher, S. W.; Briggs, R. M.; Baek, J. Y.; Lewis, N. S.; Atwater, H. A. High-Performance Si Microwire Photovoltaics. Energy Environ. Sci. 2011, 4, 866-871.

3. Yoon, J.; Baca, A. J.; Park, S.-I.; Elvikis, P.; Geddes, J. B.; Li, L.; Kim, R. H.; Xiao, J.; Wang, S.; Kim, T.-H.; et al. Ultrathin Silicon Solar Microcells for Semitransparent, Mechanically Flexible and Microconcentrator Module Designs. Nat. Mater. 2008, 7, 907-915.

4. Goodey, A. P.; Eichfeld, S. M.; Lew, K. K.; Redwing, J. M.; Mallouk, T. E. Silicon Nanowire Array Photoelectrochemical Cells. J. Am. Chem. Soc. 2007, 129, 12344-12345.

5. Spurgeon, J. M.; Boettcher, S. W.; Kelzenberg, M. D.; Brunschwig, B. S.; Atwater, H. A.; Lewis, N. S. Flexible, Polymer-Supported, Si Wire Array Photoelectrodes. Adv. Mater. 2010, 22, 3277-3281.

6. Kayes, B. M.; Atwater, H. A.; Lewis, N. S. Comparison of the Device Physics Principles of Planar and Radial P-N Junction Nanorod Solar Cells. J. Appl. Phys. 2005, 97, 114302-1-11.

7. Lou, X. W.; Zeng, H. C. An Inorganic Route for Controlled Synthesis of $\mathrm{W}_{18} \mathrm{O}_{49}$ Nanorods and Nanofibers in Solution. Inorg. Chem. 2003, 42, 6169-6171.

8. Peng, Z. A.; Peng, X. G. Nearly Monodisperse and ShapeControlled CdSe Nanocrystals via Alternative Routes: Nucleation and Growth. J. Am. Chem. Soc. 2002, 124, 33433353.

9. Ahrenkiel, S. P.; Micic, O. I.; Miedaner, A.; Curtis, C. J.; Nedeljkovic, J. M.; Nozik, A. J. Synthesis and Characterization of Colloidal InP Quantum Rods. Nano Lett. 2003, 3, 833-837.

10. Holmes, J. D.; Johnston, K. P.; Doty, R. C.; Korgel, B. A. Control of Thickness and Orientation of Solution-Grown Silicon Nanowires. Science 2000, 287, 1471-1473.

11. Ahmed, W.; Kooij, E. S.; van Silfhout, A.; Poelsema, B. Quantitative Analysis of Gold Nanorod Alignment after Electric Field-Assisted Deposition. Nano Lett. 2009, 9, 3786-3794. 
12. Mittal, M.; Furst, E. M. Electric Field-Directed Convective Assembly of Ellipsoidal Colloidal Particles To Create Optically and Mechanically Anisotropic Thin Films. Adv. Funct. Mater. 2009, 19, 3271-3278.

13. Yuan, J.; Gao, H.; Schacher, F.; Xu, Y.; Richter, R.; Tremel, W.; Muller, A. H. E. Alignment of Tellurium Nanorods via a Magnetization-Alignment-Demagnetization ("MAD") Process Assisted by an External Magnetic Field. ACS Nano 2009, 3, 1441-1450.

14. Whang, D.; Jin, S.; Wu, Y.; Lieber, C. M. Large-Scale Hierarchical Organization of Nanowire Arrays for Integrated Nanosystems. Nano Lett. 2003, 3, 1255-1259.

15. Baker, J. L.; Widmer-Cooper, A.; Toney, M. F.; Geissler, P. L.; Alivisatos, A. P. Device-Scale Perpendicular Alignment of Colloidal Nanorods. Nano Lett. 2010, 10, 195-201.

16. Redl, F. X.; Cho, K. S.; Murray, C. B.; O'Brien, S. ThreeDimensional Binary Superlattices of Magnetic Nanocrystals and Semiconductor Quantum Dots. Nature 2003, 423, 968-971.

17. Sun, S. H.; Anders, S.; Thomson, T.; Baglin, J. E. E.; Toney, M. F.; Hamann, H. F.; Murray, C. B.; Terris, B. D. Controlled Synthesis and Assembly of FePt Nanoparticles. J. Phys. Chem. B 2003, 107, 5419-5425.

18. Urban, J. J.; Talapin, D. V.; Shevchenko, E. V.; Murray, C. B. Self-Assembly of PbTe Quantum Dots into Nanocrystal Superlattices and Glassy Films. J. Am. Chem. Soc. 2006, $128,3248-3255$.

19. Clark, T. D.; Ferrigno, R.; Tien, J.; Paul, K. E.; Whitesides, G. M. Template-Directed Self-Assembly of $10 \mu \mathrm{m}$ Sized Hexagonal Plates. J. Am. Chem. Soc. 2002, 124, 54195426.

20. Knuesel, R. J.; Jacobs, H. O. Self-Assembly of Microscopic Chiplets at a Liquid-Liquid-Solid Interface Forming a Flexible Segmented Monocrystalline Solar Cell. Proc. Natl. Acad. Sci. U.S.A. 2010, 107, 993-998.

21. Dyab, A. K. F.; Ozmen, M.; Ersoz, M.; Paunov, V. N. Fabrication of Novel Anisotropic Magnetic Microparticles. J. Mater. Chem. 2009, 19, 3475-3481.

22. Smoukov, S. K.; Gangwal, S.; Marquez, M.; Velev, O. D. Reconfigurable Responsive Structures Assembled from Magnetic Janus Particles. Soft Matter 2009, 5, 1285-1292.

23. van Blaaderen, A. Colloids under External Control. MRS Bull. 2004, 29, 85-90.

24. Smith, P. A.; Nordquist, C. D.; Jackson, T. N.; Mayer, T. S.; Martin, B. R.; Mbindyo, J.; Mallouk, T. E. Electric-Field Assisted Assembly and Alignment of Metallic Nanowires. Appl. Phys. Lett. 2000, 77, 1399-1401.

25. Guihua, Y.; Anyuan, C.; Lieber, C. M. Large-Area Blown Bubble Films of Aligned Nanowires and Carbon Nanotubes. Nat. Nanotechnol. 2007, 2, 372-377.

26. Huang, Y.; Duan, X. F.; Wei, Q. Q.; Lieber, C. M. Directed Assembly of One-Dimensional Nanostructures into Functional Networks. Science 2001, 291, 630-633.

27. Hangarter, C. M.; Myung, N. V. Magnetic Alignment of Nanowires. Chem. Mater. 2005, 17, 1320-1324.

28. Keshoju, K.; Sun, L. Mechanical Characterization of Magnetic Nanowire-Polydimethylsiloxane Composites. J. Appl. Phys. 2009, 105, 023515-1-5.

29. Kayes, B. M.; Filler, M. A.; Putnam, M. C.; Kelzenberg, M. D.; Lewis, N. S.; Atwater, H. A. Growth of Vertically Aligned Si Wire Arrays over Large Areas $\left(>1 \mathrm{~cm}^{2}\right)$ with $\mathrm{Au}$ and $\mathrm{Cu}$ Catalysts. Appl. Phys. Lett. 2007, 91, 103110-1-3.

30. Yang, Z.-P.; Ci, L.; Bur, J. A.; Lin, S.-Y.; Ajayan, P. M. Experimental Observation of an Extremely Dark Material Made by a Low-Density Nanotube Array. Nano Lett. 2008, 8, 446451.

31. Kelzenberg, M. D.; Boettcher, S. W.; Petykiewicz, J. A.; Turner-Evans, D. B.; Putnam, M. C.; Warren, E. L.; Spurgeon, J. M.; Briggs, R. M.; Lewis, N. S.; Atwater, H. A. Enhanced Absorption and Carrier Collection in $\mathrm{Si}$ Wire Arrays for Photovoltaic Applications. Nat. Mater. 2010, 9, 239-244.

32. Lin, C.; Huang, N.; Povinelli, M. L. Effect of Aperiodicity on the Broadband Reflection of Silicon Nanorod Structures for Photovoltaics. Opt. Express. 2012, 20, A125-A132.
33. Lin, C.; Povinelli, M. L. Optimal Design of Aperiodic, Vertical Silicon Nanowire Structures for Photovoltaics. Opt. Express. 2011, 19, A1148-A1154.

34. Abramoff, M. D.; Magalhaes, P. J.; Ram, S. J. Image Processing with ImageJ. Biophotonics Int. 2004, 11, 36-42.

35. Plass, K. E.; Filler, M. A.; Spurgeon, J. M.; Kayes, B. M.; Maldonado, S.; Brunschwig, B. S.; Atwater, H. A.; Lewis, N. S. Flexible Polymer-Embedded Si Wire Arrays. Adv. Mater. 2009, 21, 325-328. 